\title{
C3-BIOECONOMY
}

Circular and Sustainable Bioeconomy

\section{El rol de los biocombustibles líquidos en la transición energética}

\author{
Agustín Torroba $^{1}$, Andrea Carvalho ${ }^{2}$ y Celestina Brenes Porras ${ }^{3}$
}

Autor de Correspondencia: agustin.torroba@iica.int

\section{Resumen:}

Dentro del paradigma técnico productivo de la bioeconomía, se destaca el sendero de los biocombustibles líquidos en la transición climática y energética del sector transporte. Esto debido a la posibilidad de utilización de diversos biocombustibles líquidos en reemplazo de fósiles, ya que los últimos se han constituido como una transición más limpia que permite reducir las emisiones de gases de efecto invernadero del sector, el cual tiene fuertes compromisos medioambientales. Adicionalmente, los biocombustibles líquidos serán un complemento tanto a otros paradigmas tecnológicos en desarrollo (electromovilidad, hidrógeno, etc.), como a la movilidad terrestre, aérea y marítima. En la medida que estas tecnologías atraviesan su proceso de instalación en el mercado y posterior masificación (para lo cual se requerirán varias décadas), los biocombustibles líquidos permiten avanzar en una matriz de consumo de combustibles más limpia.

Palabras clave: transición energética, biocombustibles, bioeconomía, biodiésel y bioetanol

\section{The role of liquid biofuels in the energy transition}

\author{
Agustín Torroba $^{1}$, Andrea Carvalho $^{2}$ y Celestina Brenes Porras ${ }^{3}$
}

\begin{abstract}
:
Within the productive technical paradigm of bioeconomy, the path of liquid biofuels in the climate and energy transition of the transport sector stands out. Due to the possibility of using various liquid biofuels, replacing fossils with no or few technical adaptations in the engines, these have become a cleaner transition that allows reducing greenhouse gas emissions in the sector, which has strong environmental commitments. Additionally, liquid biofuels will be a complement to other technological paradigms under development (electromobility, hydrogen, etc.), both in land, air and maritime mobility. In the meantime, while these technologies go through their installation process in the market and subsequent massification (which will require several decades), liquid biofuels allow progress in a cleaner fuel consumption matrix.
\end{abstract}

Keywords: energy transition, biofuels, bioeconomy, biodiesel and bioethanol

\footnotetext{
1 Instituto Interamericano de Cooperación para la Agricultura, IICA, Costa Rica. agustin.torroba@iica.int. CÓDIGO ORCID: https://orcid.org/0000-0001-7116-7535

2 Instituto Interamericano de Cooperación para la Agricultura, IICA. Costa Rica. andrea.carvalho.pasante@iica.int.

3 Instituto Interamericano de Cooperación para la Agricultura, IICA, Costa Rica. celestina.brenes.porras@gmail.com; CÓDIGO ORCID: https://orcid.org/0000-0001-8830-0451
}

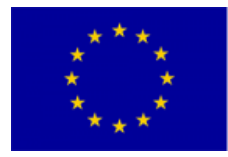

UNIÓN EUROPEA

PROYECTO COFINANCIADO POR ELFONDO EUROPEO DE DESARROLLO RES Una manera de hacer Europa

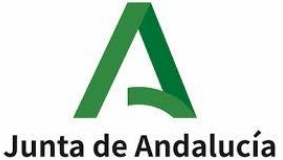
Junta de Andalucía
Andalucía
se mueve con Europa 


\section{INTRODUCCIÓN, MATERIALES Y MÉTODOS}

Desde la entrada del siglo XXI diferentes retos se han situado sobre la mesa de discusión global y el cambio climático no ha pasado desapercibido, motivo por el cual ha sido considerado desde la génesis de los Objetivos de Desarrollo del Milenio (ODM), objetivo 7 y posteriormente en los Objetivos de Desarrollo Sostenible (ODS), objetivo 6, 7, 13, 14 y 15. (Naciones Unidas, 2015 y CEPAL, 2018). Esta preocupación externalizada por la comunidad internacional ha suscitado múltiples debates en todos los continentes.

En este marco, toma fuerza un paradigma técnico productivo denominado "bioeconomía", el cual se constituye como una vía para enfrentar los desafíos del cambio climático, el deterioro de los recursos naturales y al mismo tiempo lograr beneficios tanto sociales como económicos, cumpliendo con los compromisos de reducción de GEls (gases de efecto invernadero), mientras se incrementan la producción de alimentos, fibras y energías reduciendo la dependencia en los combustibles fósiles (IICA, 2019).

Bajo este nuevo modelo de desarrollo, se han creado diferentes "senderos" de las actividades bioeconómicas. Uno de los senderos más destacados por su impacto económico y ambiental, es el de las bioenergías en general y los biocombustibles líquidos con destino al sector transporte en particular.

Cabe destacar que para el año 2014 el sector de transporte aportó más del $14 \%$ de las emisiones de CO2 equivalente del mundo (IPCC, 2014) incrementando la presión sobre el calentamiento global y sus efectos adversos.

El presente trabajo pretende analizar el rol del consumo y producción sustentable de biocombustibles líquidos (entendidos como aquellos productos bioenergéticos que aportan a la descarbonización del sector transporte) en la transición energética hacia un mundo alineado con las necesidades de reducir las emisiones de gases de efecto invernadero.

El enfoque de la investigación se caracteriza por ser mixto debido a la naturaleza compleja del fenómeno de la investigación, combinando tanto los 
elementos cualitativos y cuantitativos para obtener una mejor exploración y explotación de los datos. (Hernández-Sampieri, 2014, p.532-533).

De esta forma, se recurre a fuentes secundarias como revisión bibliográfica de documentos, informes referentes a la temática y series de datos estadísticos. A partir de ellos, se utilizan instrumentos tales como gráficos, los cuales tienen como fin mostrar la conducta de los biocombustibles líquidos y, de esta manera, se establecen las tendencias o comportamientos estacionales (Nussbaumer, 2015) con el objetivo de tener un panorama amplio sobre la temática que permita arribar a conclusiones sobre el rol de los biocombustibles líquidos en la transición energética.

En ese sentido el documento se divide en tres secciones, la presente, donde se introduce a la problemática y método. La segunda muestra la necesidad en la que se enmarca el cambio de paradigma de matrices energéticas dependientes a combustibles fósiles hacia un paradigma más sostenible, a la vez que analiza tanto la situación actual como las proyecciones de los biocombustibles líquidos en diferentes medios de transportla última culmina con las principales discusiones y conclusiones alrededor de la temática.

\section{RESULTADOS}

\subsection{Nuevos paradigmas de movilidad y transición limpia}

El sector de transporte es el cuarto emisor de gases de efecto invernadero, concentrando el $74 \%$ de sus emisiones en vehículos de pasajeros y de carga por carretera, los cuales utilizan principalmente gasolina y diésel como combustibles. Mientras que el subsector de aviación y marítimo emiten un 11\% del total (cada uno), quedando el $3 \%$ en los subsectores ferroviarios y otros (IEA 2020).

El nivel actual de emisiones del sector transporte, dificulta el cumplimiento del Acuerdo de Paris (NFCCC, 2015), el cual indica como principal punto la necesidad internacional de limitar en el siglo XXI el aumento global de la 
temperatura a $2{ }^{\circ} \mathrm{C}$ y, al mismo tiempo, buscar medios para frenar el aumento de la temperatura a $1,5^{\circ} \mathrm{C}$ con respecto a las eras preindustriales.

Bajo este contexto de demandas medioambientales, el actual paradigma de movilidad vehicular terrestre, basado en el uso de motores de combustión interna, intensivos en el uso de combustibles fósiles, especialmente gasolina y diésel se encuentra en tensión, frente a la aparición de nuevas alternativas tecnológicas más limpias, principalmente la electromovilidad y el uso de hidrógeno.

En efecto, estas innovaciones en el sector automotriz responden a las urgentes necesidades ambientales, no obstante, realizar la migración masiva hacia algunas de estas alternativas pueden tomar varias décadas (IRENA 2020 e IEA 2020), lo que resulta problemático en virtud de los compromisos ambientales asumidos en un corto plazo para reducir los GEls. Por ello, resulta menester ejecutar una transición sustentable pero efectiva y eficiente en el corto y mediano plazo.

En ese sentido, los biocombustibles líquidos, en el formato de biodiésel y bioetanol4, han comenzado a liderar una transición más limpia5, dentro del paradigma de movilidad actual, motivado por la formulación de políticas públicas que se asientan, principalmente, en alguno de los siguientes pilares: i) los aspectos medioambientales y de salud humana;; ii) la seguridad y diversificación energética; y, iii) el desarrollo agrícola y las ventajas económicas derivadas de la producción y uso de biocombustibles.

Cabe destacar que el rol de los biocombustibles líquidos también permitirá complementar nuevos paradigmas de movilidad en el medio y largo plazo. En tal sentido, debido a restricciones en la densidad energética de las

\footnotetext{
4 El biodiésel y bioetanol son combustibles biológicos que se utilizan como complemento o sustituto del diésel y gasolina fósil. En el 2019 sustituyeron el consumo, en términos volumétricos, del 5,5\% de la gasolina y el 3,1\% del diésel mundial (Torroba 2021 a).

5 Sobre la reducción de emisiones de GEls de los biocombustibles líquidos en comparación a los combustibles fósiles, puede consultarse IPCC (2014) y Torroba (2020 a). Para mayor detalle sobre el rango de reducción de emisiones de gases de efecto invernadero.

${ }^{6}$ Las políticas públicas de biocombustibles también suelen establecer requisitos mínimos de reducción de emisiones de GEls y clasificaciones alternativas en virtud de ello y otras características (biocombustibles convencionales, avanzados, etc.)
} 
baterías, y la falta de una infraestructura en formato de redes o estaciones de carga masiva, la electromovilidad7 aumentaría su inserción en automóviles de tamaño pequeño para distancias cortas, así como en vehículos de dos y tres ruedas. Otros tipos de tecnologías con menores necesidades de recarga, como el hidrógeno, tendrían un mayor grado de penetración en vehículos pesados y de larga distancia (camiones y buses) mientras que, en simultáneo, las transiciones limpias (biocombustibles, vehículos híbridos, híbridos enchufables, etc.) convivirían en la gama de automóviles de pasajero de tamaño y distancias medias (IRENA 2020 y Torroba 2021 b).

Por otro lado, en cuanto a otros sectores del transporte, como la navegación, pero especialmente la aviación, se observa que, debido a la complejidad de sus sistemas, resultará efectivo el uso biocombustibles líquidos ante la ausencia de alternativas de movilidad más limpias a corto y medio plazo (IEA, 2021). En tal sentido, en 2010 la Organización de Aviación Civil Internacional (OACl, 2013) externó su preocupación sobre la alta cantidad de emisiones de gases de efecto invernadero producidas por la aviación civil internacional, motivo por el cual decidió realizar una seria de metas que apuntan a reducir sus emisiones de carbono en un 50\% para 2050 en comparación con los niveles de 2005. (Torroba, 2021 a).

La Agencia Internacional de Energía (IEA, 2019), considera que, para cumplir con este objetivo, se deberá mezclar biocombustibles líquidos de aviación con combustible fósil para aviones, debido a que los primeros son "la única opción actual para reducir significativamente las emisiones del sector de aviación".

\footnotetext{
7 Adicionalmente, la electromovilidad enfrenta otros desafíos. El más destacado es que la electricidad producida para los vehículos provenga de fuentes limpias para evitar un aumento en las emisiones de gases de efecto invernadero a lo largo de todo el ciclo de vida. En este sentido, el $64 \%$ de la electricidad producida en el mundo, proviene de fuentes fósiles, siendo el carbón su fuente de energía más contaminante y la más utilizada en la generación de electrones, con un 38\% del total mundial (Torroba 2021 a).

8 Asimismo, hay en estudio tecnologías disruptivas que utilizan a los biocombustibles como insumo, destacándose la Celda de Combustible de Óxido Sólido (SOFC). En esta tecnología, la celda de combustible se alimenta con bioetanol para generar electricidad. El sistema posee un reformador catalítico que libera el hidrógeno contenido en el biocombustible, que luego se convierte en electricidad en un dispositivo electroquímico, que es la celda propiamente dicha.
} 
De manera análoga, la Organización Marítima Internacional (OMI, 2018) creó una estrategia concreta para evitar que la contaminación marítima se acrecenté y de modo contrario, esta sea controlada y reducida, dando paso a la posible introducción de biocombustibles en el sector para reducir las emisiones de gases de efecto invernadero y de azufre.

En el capítulo siguiente, se describirá cómo se han insertado los biocombustibles líquidos, representando hoy una transición más limpia a los fósiles y sus perspectivas a futuro, con especial énfasis en usos que hoy son marginales, como los segmentos de aviación y navegación.

\subsection{Evolución reciente de los biocombustibles líquidos y perspectivas}

Como establece Torroba (2021 a), la producción y el consumo de biocombustibles líquidos a nivel mundial, ha mostrado una importante expansión en los últimos veinte años. Con relación al consumo de biocombustibles líquidos a nivel mundial, se evidencia un crecimiento acumulado del 47 \% en los últimos 10 años.

En 2019 el consumo superó los 163 millones de metros cúbicos, en el año 2020, las medidas de confinamiento implementadas a causa de la pandemia, con la consecuente reducción de la movilidad, generaron una reducción interanual del -10 \%. En el caso del bioetanol, el efecto fue más fuerte, presentando una reducción interanual de -15\%. En lo que respecta al biodiésel, solo se observó una reducción en su tasa de crecimiento, aumentando un 1.5 $\%$, interanual.

Se observa que la evolución de la producción y consumo de los biocombustibles está fuertemente influenciada por la formulación de políticas públicas implementadas por los países. Uno de los instrumentos más utilizados para promover su consumo son los denominados "mandatos" de uso de biocombustibles, los cuales establecen la obligación de mezclar biocombustibles con combustibles fósiles, fijando una cuota de mercado. Si bien estos no siempre son cumplidos en su totalidad, suelen representar la mayor parte del consumo de los países. 
Según Torroba (2020 b), se registraron al menos 53 mandatos de uso de bioetanol en 2019. Por otro lado, se destaca la presencia de mercados libres de amplio desarrollo, como el caso de Brasil en donde se comercializa bioetanol hidratado, o el E85 en Estados Unidos y varios países de Europa. En el caso del biodiésel, en 2019 se contabilizaron 43 países con mandato.

En sintonía con la implementación de diferentes tipos de políticas públicas para promover el consumo de los biocombustibles, se proyecta una recuperación en la producción de biocombustibles a los niveles anteriores a la pandemia, durante el transcurso del 2021. De acuerdo con el informe OCDE/FAO (2020), este aumento se dará principalmente en los países en desarrollo, impulsado fundamentalmente por la aplicación de mandatos con mayores tasas de mezcla. También se prevé una recuperación por ampliación de políticas públicas en países en desarrollo?.

En el mediano y largo plazo, será necesario incrementar fuertemente la producción mundial de biocombustibles para poder cumplir los compromisos medioambientales fijados. De acuerdo con IRENA (2020), se fija un escenario denominado "Planned Energy Scenario"10, en el cual la producción de biocombustibles se incrementaría un $110 \%$ al 2030 (en comparación con 2017), alcanzando un 189 \% en 2050. En este mismo informe, se establece un escenario de descarbonización más exigente denominado "Transforming Energy Scenario"11, en el cual se incrementaría la producción a un 379 \% en 2050, donde un $15 \%$ se espera sean biocombustibles de aviación.

\footnotetext{
- Se destaca el caso de Indonesia, que expandió en el 2021 su mandato de uso de biodiesel al 30\% o B30, pero tiene como objetivo expandirlo, pasando a un B40 en el 2022. En relación con las Américas, Brasil continúa con su programa de ampliación de mandato, el cual busca aumentar un punto porcentual por año hasta llegar a un B15 en 2023. También, el caso de Colombia, el cual avanzó en la aplicación de B12 partir de abril del 2021 en casi todo el país.

10 Es el caso de referencia principal para el citado estudio, proporcionando una perspectiva sobre el desarrollo del sistema energético basado en planes energéticos actuales de los gobiernos y otros objetivos y políticas planificados (a partir de 2019), incluidas las contribuciones determinadas a nivel nacional (NRD)en virtud del Acuerdo de París a menos que el país tenga objetivos o planes climáticos y energéticos más recientes

11 El mencionado escenario describe una ambiciosa, pero realista, vía de transformación energética basada en gran medida en fuentes de energía renovables y eficiencia energética constantemente mejorada (aunque no se limita exclusivamente a estas tecnologías). Esto pondría al sistema energético en el camino necesario para mantener el aumento de las temperaturas globales a muy por debajo de 2 grados Celsius $1^{\circ}$ C) y hacia $1,5^{\circ} \mathrm{C}$ durante este siglo.
} 
Por otra parte, en un reciente informe publicado por IEA (2021), se describe cómo el sector energético global puede llegar a un escenario de cero emisiones netas en el año 2050. Para lograr dicha meta, se prevé un fuerte incremento en el consumo de los biocombustibles, en la presente década, con un aumento del $275 \%$, como se menciona en la figura 1.

Cabe destacar, que este aumento estará fuertemente concentrado en la movilidad terrestre por carretera. A partir del 2030, el consumo seguiría creciendo, pero de forma más lenta y ocurrirá un re-direccionamiento hacia nuevos sectores, como son los biocombustibles de aviación y marítimos.

Figura 1: Posible evolución del consumo de biocombustibles líquidos (en millones de metros cúbicos) de acuerdo con el escenario de cero emisiones netas para el 2050 de la IEA.

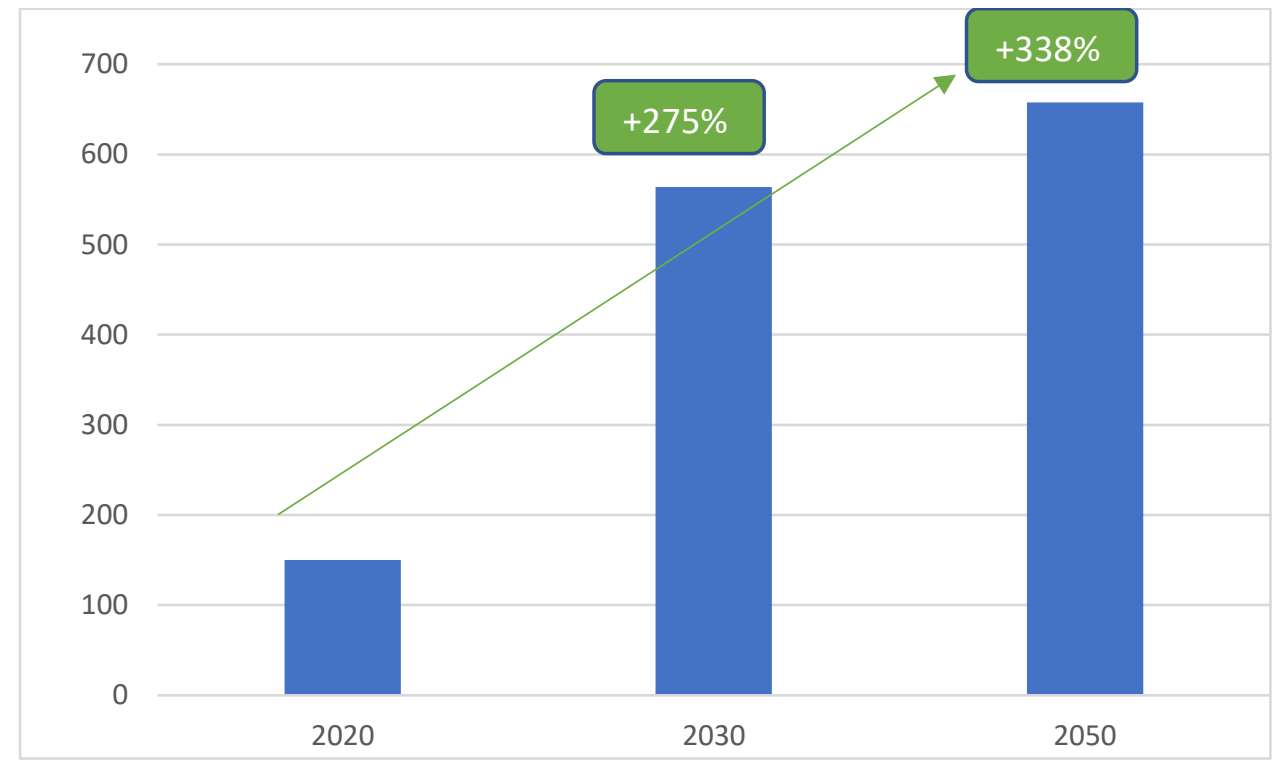

Fuente: Torroba (2021b).

Un escenario menos exigente en materia de emisiones, pero en correspondencia con los compromisos establecidos en el Acuerdo de París, es el denominado "Escenario de Desarrollo Sostenible", establecido por la IEA (2020), en el mismo, se espera que la producción de biocombustibles líquidos se incremente un $210 \%$ en el 2030 tomando como referencia el consumo de 2019 , llegando a los 298 millones de toneladas equivalentes de petróleo.

Este incremento, estará dado principalmente por el bioetanol y el biodiésel utilizado por el transporte terrestre, en donde el $56 \%$ se consumirían 
como biocombustibles destinados al transporte de pasajeros y el $28 \%$ al transporte de mercancías por carretera. Por otro lado, se espera el ingreso de otros sectores, con un $10 \%$ del consumo dedicado a la aviación, mientras que $5 \%$ correspondería al transporte marítimo.

\section{DISCUSIÓN / CONCLUSIONES}

Los compromisos ambientales, en virtud de la necesidad de reducir emisiones de gases de efecto invernadero, pero también en la mejora de la calidad del aire, vienen poniendo presión al sector de transporte terrestre, marítimo y aéreo, por el uso intensivo de combustibles fósiles.

Bajo los nuevos requerimientos, se impone la necesidad de establecer una transición ambientalmente más limpia en el sector, donde emergen con fuerza el consumo de biocombustibles líquidos. Especialmente en el transporte terrestre, impulsados por el consumo de biodiésel y el bioetanol, los biocombustibles han tenido una fuerte expansión en los últimos 20 años y se han instalado con fuerza en la matriz de combustibles líquidos, permitiendo contar con combustibles de origen biológico y de menores emisiones de GEls. Ello ha avanzado fuertemente por la formulación de políticas públicas en los distintos países alrededor del mundo.

Asimismo, la búsqueda de una movilidad de menores emisiones está generando nuevos paradigmas, basados en tecnologías como la electromovilidad y el hidrógeno, las cuales se están comenzando a expandir. Sin embargo, se estima que la masificación de estos nuevos paradigmas podría tardar varias décadas, por lo cual los biocombustibles líquidos jugarán un rol crucial en la descarbonización del subsector de transporte terrestre en la transición. Asimismo, cumplirá un rol complementario en el futuro, al quedar nichos de mercado que posiblemente no puedan ser cubiertos por nuevas tecnologías.

En línea con las necesidades ambientales, los distintos escenarios plantean la necesidad de que los biocombustibles líquidos con destino terrestre 
aumenten fuertemente su consumo al 2050. La discusión sobre el rol de los biocombustibles líquidos en la transición climática gira principalmente en torno a los aspectos cuantitativos (qué cantidad será necesaria producir y consumir para cumplir los compromisos medioambientales) y la distribución relativa de los diferentes tipos de biocombustibles por subsector.

Finalmente, otros destinos, hoy marginales como los biocombustibles marinos, pero especialmente los destinados a la aviación, tendrán un rol destacado ante la ausencia de nuevas tecnologías que permitan descarbonizar estos subsectores. Por ello, el consumo de biocombustibles de aviación y marítimos se expandirán al 2040, y lo harán con mucha más velocidad entre el 2040 y 2050, observándose también un redireccionamiento de materias primas y biocombustibles terrestres a estos destinos.

\section{REFERENCIAS}

CEPAL. (Comisión Económica para América Latina y el Caribe) (2018). La Agenda 2030 y los Objetivos de Desarrollo Sostenible Una oportunidad para América Latina y el Caribe. https://repositorio.cepal.org/bitstream/handle/11362/40155/24/S1801141 es.pdf

UNFCCC (United Nations Climate Change) (2015). Convención Marco de las Naciones Unidas sobre el Cambio Climático: Acuerdo de París. https://unfccc.int/sites/default/files/spanish_paris_agreement.pdf

HERNÁNDEZ-SAMPIERI, R. (2014). Metodología de la Investigación (6ta Edición ed.). México D.F, McGraw-Hill Education.

IEA (International Energy Agency, Francia). (2019). Indicadores de transición energética. Recuperado de: https://www.iea.org/reports/energytransitions-indicators

IEA (International Energy Agency) . (2020). Tracking transport. Recuperado de: https://www.iea.org/topics/transport 
IEA (International Energy Agency). (2021). Net Zero by 2050 A Roadmap for the Global Energy Sector. Recuperado de: https://www.iea.org/events/netzero-by-2050-a-roadmap-for-the-global-energy-system

IICA (Instituto Interamericano de Cooperación para la Agricultura, Costa Rica). (2019). Programa de Bioeconomía y Desarrollo Productivo: Abordajes conceptuales y metodológicos para la cooperación técnica. San José, Costa Rica, IICA.

IPCC (Intergovernmental Panel on Climate Change. Switzerland). (2014). Climate Change 2014: Synthesis Report. Contribution of Working Groups I, II and III to the Fifth Assessment Report of the Intergovernmental Panel on Climate Change. Recuperado de: https://www.ipcc.ch/report/ar5/syr/

IRENA (International Renewable Energy Agency). (2020). Global Renewables Outlook: Energy 2050. https://www.irena.org/publications/2020/Apr/Global-RenewablesOutlook-2020

Naciones Unidas. (2015). Objetivos de Desarrollo del Milenio Informe de 2015. Recuperado de: https://www 1.undp.org/content/undp/es/home/librarypage/mdg/themillennium-development-goals-report-2015.html

NUSSBAUMER, C. (2015) Storytelling with data. Canada, Wiley.

OCDE/FAO. (2020). OCDE-FAO Perspectivas Agrícolas 2020-2029. Recuperado de: https://doi.org/10.1787/a0848ac0-es

OACI (Organización de Aviación Civil Internacional). (2013). Informe anual del Consejo. Recuperado de: https://www.icao.int/publications/Documents/10001 es.pdf

OMI (Organización Marítima Internacional). (2018). IMO Action to Reduce Greenhouse Gas Emissions from International Shipping. https://sustainabledevelopment.un.org/content/documents/266201MO 
ACTION TO REDUCE GHG EMISSIONS FROM INTERNATIONAL SHIPPING. pdf

TORROBA, A. (2020 a). Los biocombustibles líquidos en las Américas: situación actual y potencial de desarrollo. San José, Costa Rica, IICA. Recuperado de:

https://repositorio.ïca.int/bitstream/handle/1 1324/9975/BVE20058034e.p df? sequence $=1$ \&isAllowed $=y$

TORROBA, A. (2020 b). Atlas de los biocombustibles líquidos 2019-2020. San José, Costa Rica, IICA. Recuperado de: https://repositorio.iica.int/bitstream/handle/11324/13974/BVE20128304e. pdf? sequence $=1$ \&isAllowed $=y$

TORROBA, A. (2021 a). Atlas de los biocombustibles líquidos 2020-2021. San José, Costa Rica, IICA. https://repositorio.iica.int/bitstream/handle/11324/18661/BVE21097939e. pdf? sequence $=1$ \&isAllowed $=y$

TORROBA, A. (2021b). Biocombustibles líquidos. Institucionalidad y formulación de políticas públicas. San José, Costa Rica, IICA. Recuperado de: http://repositorio.iica.int/handle/11324/18566

VOHRA, K.; VODONOS, A.; SCHWARTZ, J.; MARAIS, E.A.; SULPRIZIO, M.P.; MICKLEY, L.J. (2021). Global mortality from outdoor fine particle pollution generated by fossil fuel combustion: Results from GEOS-Chem, Environmental Research, Vol (195), p.24-51 https://doi.org/10.1016/i.envres.2021.110754. 